\title{
MULTISPECTRAL IMAGE CAPTURING WITH FOVEON SENSORS
}

\author{
R. Gehrke*, A. Greiwe \\ University of Applied Sciences Frankfurt am Main, Department of Architecture, Civil Engineering and Geomatic,
Germany
(ralf.gehrke, ansgar.greiwe)@fb1.fh-frankfurt.de
}

Commission I, ICWG I/5

KEY WORDS: UAV, remote sensing, Foveon, geometric calibration

\begin{abstract}
:
This article describes a specific image quality problem using an UAV and the commercially available multispectral camera Tetracam ADC Lite. The tests were carried out with commercially available UAV Multirotor MR-X 8 performed under normal use and conditions. The ADC Lite shows a remarkable rolling shutter effect caused by the movement and vibrations of the UAV and a slow readout speed of the sensor.

Based on these studies the current state of a sensor development is presented, which is composed of two compact cameras with Foveon sensors. These cameras allow to record high quality image data without motion blur or rolling shutter effect. One camera captures the normal colour range; the second camera is modified for the near infrared.

The moving parts of both cameras are glued to ensure that a geometric camera calibration is valid over a longer period of time. The success of the gluing procedure has been proven by multiple calibrations. For the matching of the colour- and infrared image the usability of calibrated relative orientation parameters between both cameras were tested. Despite absolutely synchronous triggering of the cameras by an electrical signal, a time delay can be found up to 3/100 s between the images. This time delay in combination with the movement and rotation of the UAV while taking the photos results in a significant error in the previously calibrated relative orientation. These parameters should not be used in further processing.
\end{abstract}

This article concludes with a first result of a 4-channel image and an outlook on the following investigations.

\section{INTRODUCTION}

Mini-UAV with a maximum take-off weight up to $5 \mathrm{~kg}$ close the gap between terrestrial-based measurements and data acquisitions carried out by manned aircraft. The usual payload of these aircrafts is up to $1.5 \mathrm{~kg}$. However, a reduction of payload is aimed for the benefit of a longer flight-duration and steadier flight characteristics. For the production of 3D-models and orthorectified images SLR and high-quality compact cameras are commonly used. For data processing software packages of the conventional aerial imagery as well as computer vision are used.

For aerial photogrammetry with UAV, the use of optical sensors in the near infrared (700 nm - $1000 \mathrm{~nm})$ is less common. Typical applications for these sensors are agriculture (Berni et al. 2009), environmental protection and also the aerial archaeology (Verhoeven et al. 2009). For this purpose, a few sensors for UAV are already commercially available. Lightweight sensors for light aircraft and UAVs such as the Tetracam ADC (Huang et al.) have been modified by the manufacturer once again in favour of a lower weight. The model Tetracam ADC Lite that emerged from this optimization is presented in this article.

In academia such sensors are realized by the modification and usage of appropriate filters on conventional RGB (red green blue)-cameras (Gini et al. 2012) or also on panchromatic industrial cameras (Nebiker et al. 2008). In most cases the spectral selectivity is under-utilised due to the usage of a bandpass filter outside the visible range. A 1-channel image instead of a 3-channel (RGB) image is created. Alternative methods preserve the spectral selectivity of the colour sensor. The red, green, or blue light is blocked by a band-stop filter, the sensitivity of these pixels for the infrared light is used to calculate an infrared channel (Tetracam 2010, Knoth et al. 2011). The mathematical model of the pinhole camera (central projection) is assumed for the images in the geometric processing. When using computer vision software it's possible to calculate the cameras interior orientation for each image. For the use of conventional aerial photogrammetry software a stable interior orientation over a longer period of time is required. The stability of a camera calibration can be examined through multiple calibrations (Rieke Zapp et al., 2010). For automatic image matching algorithms during the processing, high quality, sharp recorded images are required (Greiwe \& Gehrke 2013).

The objective of this paper is to show the results of an examination of the image quality of a commercially available UAV sensor under real world conditions. Based on this, the current state of an in-house developed sensor head for miniUAV is presented. This sensor produces high quality 4-channel imagery. The current state of development includes the sensor design and first investigations into the camera geometry. The

\footnotetext{
* Corresponding author.
} 
paper concludes with first results and an outlook on further investigations.

\section{UAVS LIMITING FACTORS}

As sensor platform the octocopter Multirotor MR-X8 was used. It is characterized by a maximum payload of about $1500 \mathrm{~g}$ at a maximum take-off weight of $3.5 \mathrm{~kg}$. The UAV has a modular structure. Different sized batteries can be combined with different payload weights. The flight attitude is automatically controlled by a series of sensors. They control an actively updated two-axis stabilised suspension of the payload, which can to some extent compensate for the UAVs rotation. However, several years of experiences of the authors showed that exposure times of maximum $1 / 1000 \mathrm{~s}$ are necessary to get images without motion blur. These short exposure times require a fast lens and the possibility of a subsequent editing of the images in raw format (Verhoeven 2010).

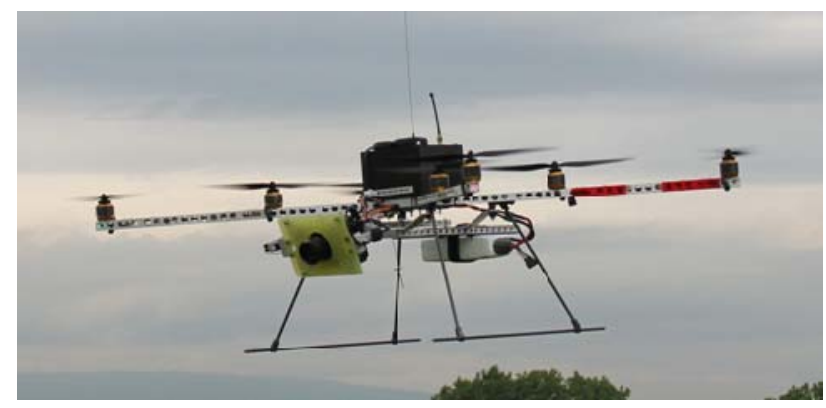

Figure 1. MR-X8 with Tetracam ADC Lite

\section{TETRACAM ADC LITE}

The Tetracam ADC Lite is a lightweight (200 g) multi spectral camera that records the wavelength ranges of green, red and near infrared light. It is equipped with a sensor with color filtering by a Bayer-pattern. The manufacturer replaced the built-in hotmirror by a band-stop filter blocking the blue light. The blue-sensitive pixels of the Bayer-pattern are sensitive for the infrared light too. Correction terms for the red and green pixels can be calculated with the manufactures software Pixelwrench2. A green, red, infrared image is created (Fig. 2). More data can be found in table 3.

In the field the ADC Lite was used with the MR-X8 and a twoaxis stabilised suspension. In the image data a rolling shutter effect can be observed (fig. 2), which is caused by a slow readout speed of the sensor. This effect is primarily dependent on the vibration and rotation rate existing at the time of the recording. In turn these are dependent on weather conditions. In perfect weather conditions (no wind) approximately every second image taken by the Tetracam shows this effect. At stronger winds up to $5 \mathrm{~m} / \mathrm{s}$ (limit for UAV operations by law) almost every image has a rolling shutter effect (fig. 2 down). This effect leads to deviations from the mathematical model of the central projection. Such variations can be modelled (Geyer et al. 2005); but they are not implemented in conventional photogrammetric software products. The manufactures software Pixelwrench2 also does not provide any functionality to correct the data.
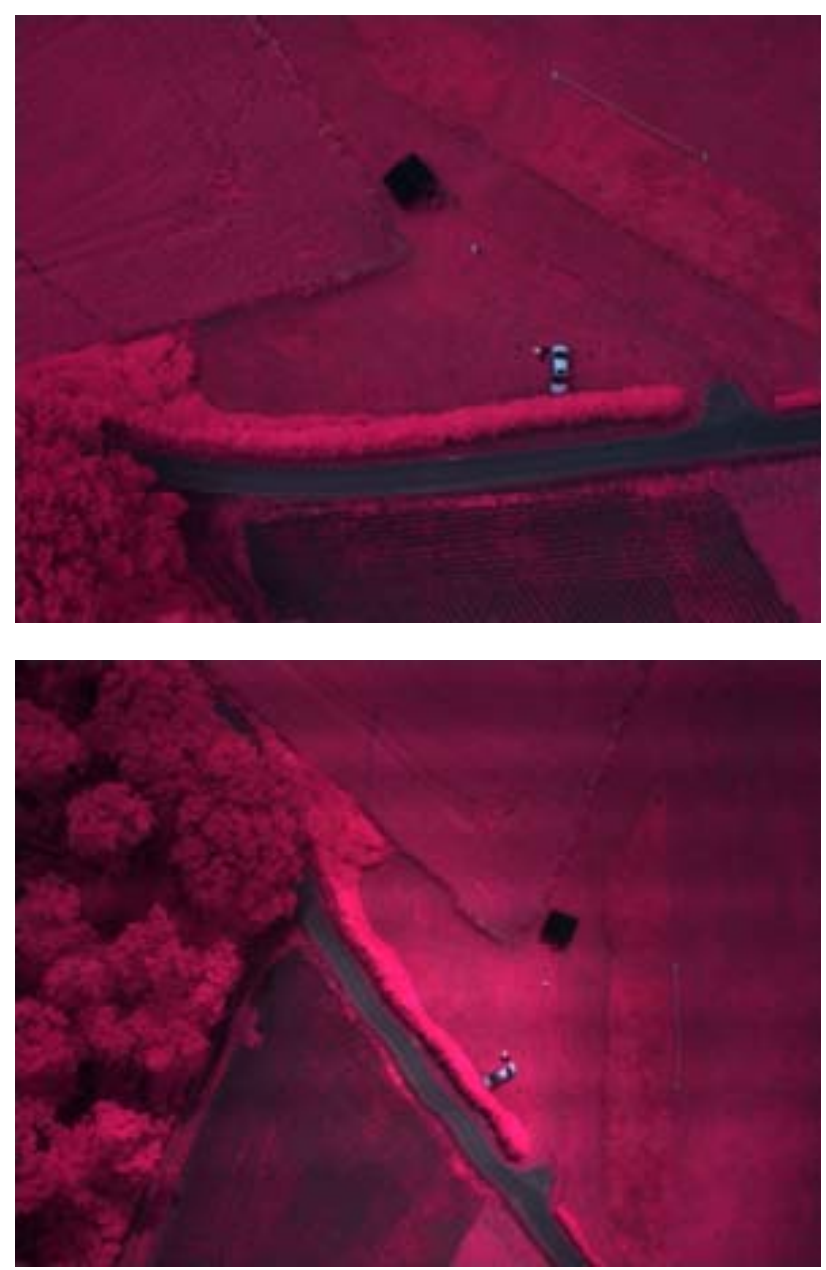

Figure 2. ADC images with no (top) and with rolling shutter effect (down)

\begin{tabular}{|l|l|l|l|l|}
\hline & Weight & $\begin{array}{l}\text { Focal } \\
\text { length }\end{array}$ & Sensor size & Pixel \\
\hline $\begin{array}{l}\text { Sigma } \\
\text { DP1 }\end{array}$ & $\begin{array}{l}\text { each } \\
\text { approx. } \\
250 \mathrm{~g}\end{array}$ & $16,6 \mathrm{~mm}$ & $\begin{array}{l}20,7 \times \mathrm{x} 13,8 \\
\mathrm{~mm}^{2}\end{array}$ & $\begin{array}{l}2640 \\
\mathrm{x} \\
1760\end{array}$ \\
\hline $\begin{array}{l}\text { Tetracam } \\
\text { ADC Lite }\end{array}$ & $\begin{array}{l}\text { approx. } \\
200 \mathrm{~g}\end{array}$ & $8,5 \mathrm{~mm}$ & $\begin{array}{l}6,59 \times \mathrm{x} \\
\mathrm{mm}^{2}\end{array}$ & $\begin{array}{l}2048 \\
\mathrm{x} \\
1536\end{array}$ \\
\hline
\end{tabular}

Table 3. Technical data of the sensors

\section{CONSTRUCTION AND INVESTIGATION OF A SIGMA DP1 SENSOR HEAD}

\subsection{Construction}

The Sigma DP1 is a compact camera with a wide-angle lens, raw data logging and a FOVEON X3 sensor. The outstanding image quality of this camera when used on UAVs (Gehrke \& Greiwe 2011) and its technical background (Gilblom et al. 2003) have already been described. The most important technical data are summarized in table 3 .

By removing the built-in hotmirror (fig. $4,2^{\text {nd }}$ from left) the sensor sensitivity for the near infrared light up to approx. $1000 \mathrm{~nm}$ is used (Gilblom \& Yoo 2004). An infrared filter Hoya R720 is used for the separation of the infrared light from 
the remaining spectrum. Combined with a second Sigma DP1 without infrared conversion the capture of a 4-channel image with blue, green, red and near infrared channels is possible.

For the use of conventional aerial imagery software, both cameras are stabilized and geometrically calibrated using the spatial testfield at the University of Applied Sciences in Frankfurt/Germany. For the stabilization process, both cameras are focused in their respective spectral range at a distance of $75 \mathrm{~m}$ and all parts of the retractable lens are completely glued.
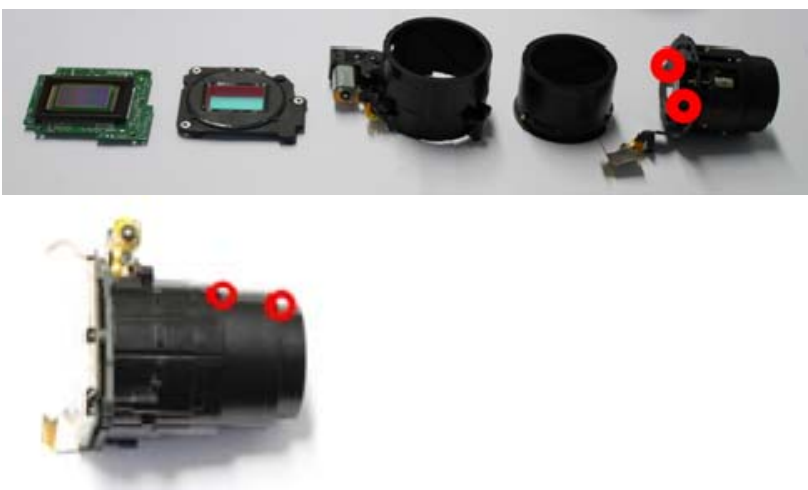

Figure 4. Gluing points at the Sigma DP1 lens

Both cameras are aligned parallel on a frame. The simultaneous shutter release is possible by a tap in the cameras electronics. For this task the shutter release contactors of both cameras are switched parallel. This ensures that the trigger pulse reaches both cameras at the same time.

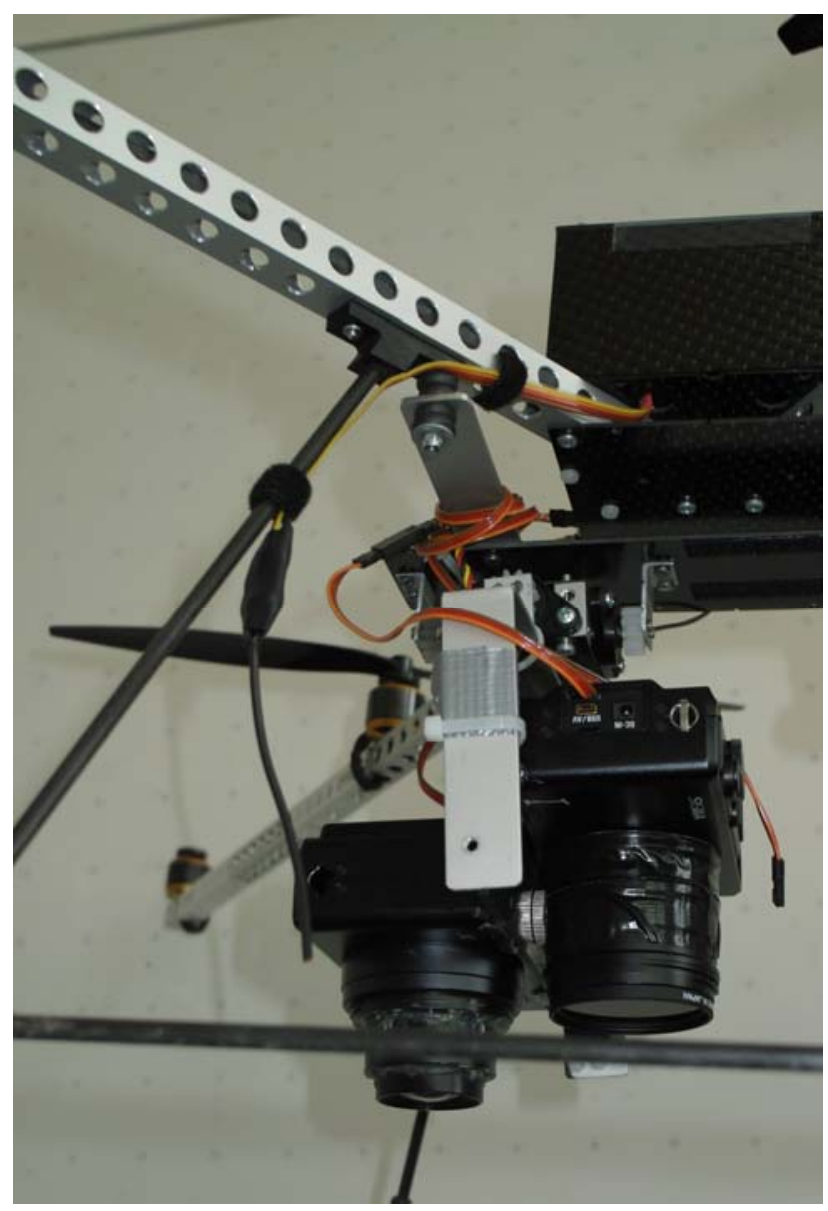

Figure 5. Sensor head with RGB and IR camera
The exposure adjustment is carried out on site manually. An adaption of the exposure afterwards is possible by imaging a spectral reference (Spectralon) on site before and after the flight. By freely available raw data converters, it is possible to generate image data that contains no "photographic adjustments" such as white balance and gamma correction.

\subsection{Geometric calibration}

The success of the camera gluing should be confirmed by the investigation of the stability of the interior orientation parameters. The study of Rieke-Zapp et al. (2010) for a not stabilized Sigma DP1 serves as a reference.

For this task, the camera calibration is perfomed 5 times with switching the cameras on and off between the calibrations. For the calculation of the interior orientation parameters the software Pictran is used. All parameters, such as principal distance, principal point, radial-symmetric distortion, decentring distortion, affinity and shear are calculated. The two most important parameters principal distance and principal point are shown in figure 6 and 7.

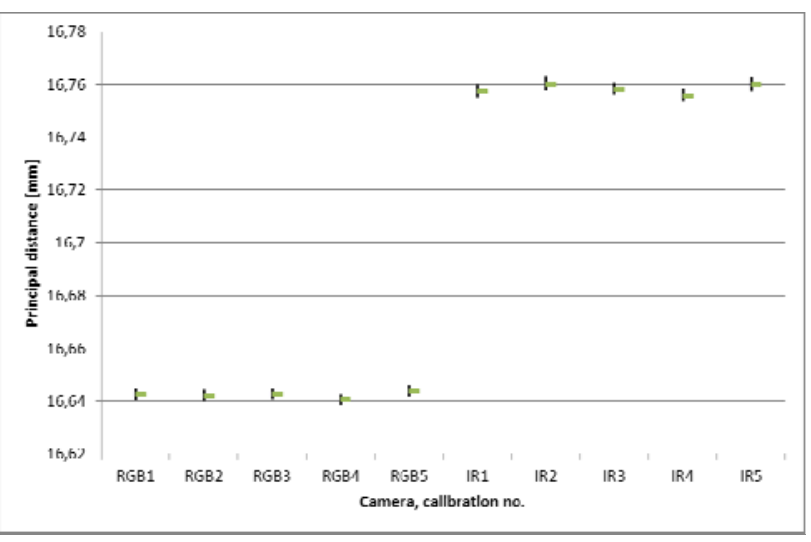

Figure 6. Calibration results for principal distance with the confidence interval $(2 \sigma, 95 \%)$ as black line 


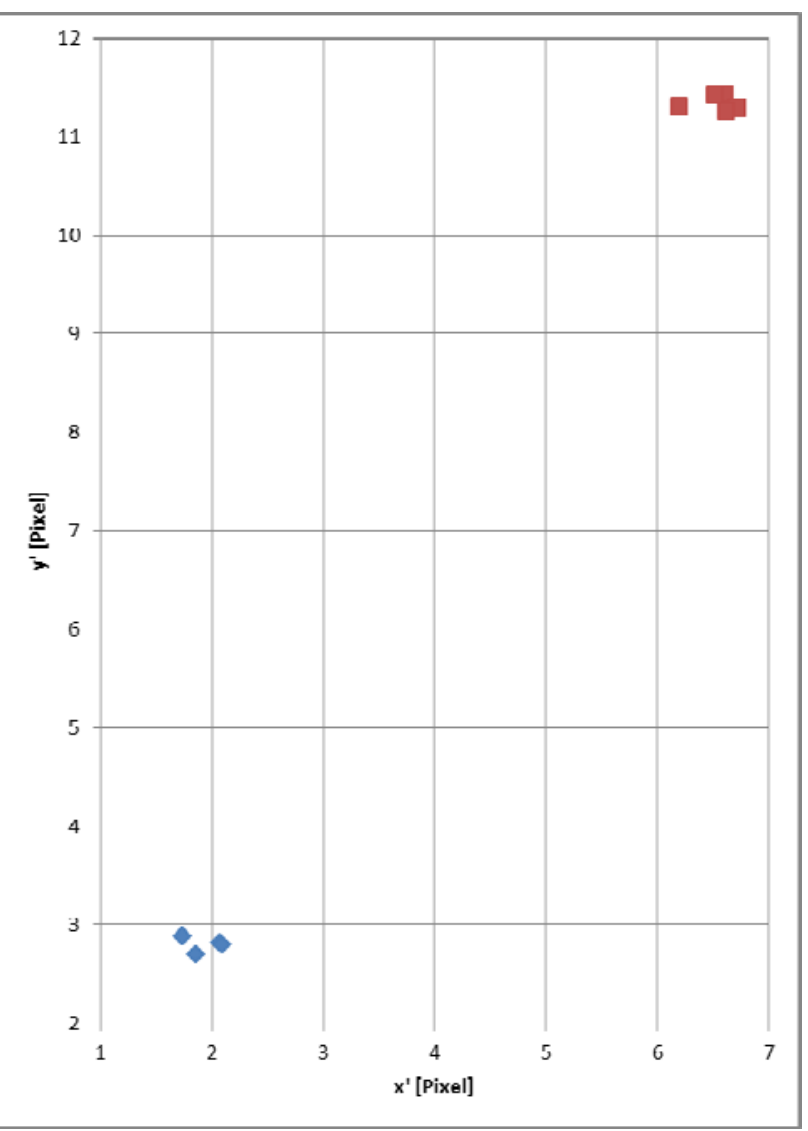

Figure 7. Calibration results for principal point of the RGB camera (blue) and the IR camera (red)

The principal distance of both cameras is assumed to be stable regarding the confidence interval of $95 \%(2 \sigma)$. The stability of the principal point of an unstabilized Sigma DP1 was examined by Rieke-Zapp et al. (2010) with an uncertainty of principle point of several pixels. The cameras principal points in this investigation vary below one pixel and are assumed to be stable.

The proven stability ensures the usage of the camera calibration over a longer period of time. However, for quality assessment a camera calibration before and after a mission is recommended.

The exterior orientation parameters are also determined in a calibration process. With these parameters, an orientation of the cameras to each other can be calculated. The interior precision of this orientation is about $1 / 10 \mathrm{~mm}$ in the position and $2 / 100$ to 9/100 degree in the alignment. This can be used for the following image matching of the RGB- and IR-image. But it is imperative that both cameras are triggering at absolutely the same time. Otherwise the movement and rotation of the UAV during the image capturing makes these parameters obsolete.

\subsection{Simultaneous shutter release}

To study synchronous triggering 20 photos of an analogue stopwatch are taken with a shutter speed of $1 / 1000 \mathrm{~s}$. The scale of the stopwatch has a resolution of $1 / 100 \mathrm{~s}$. Although the cameras are absolutely synchronously triggered by an electrical signal, a delay can be found between the individual images ranging from $-3 / 100 s$ to $+3 / 100 s$ with a standard deviation of $13 / 1000 \mathrm{~s}$. A systematic error is not discernible. At a typical UAV velocity of $3 \mathrm{~m} / \mathrm{s}$ the position error between both cameras would be $9 \mathrm{~cm}$. The angular velocity is required for an error estimation of the orientation between both cameras at release time stamp. Because the angular velocity is unknown, it is estimated by the maximum shutter speed $1 / 1000 \mathrm{~s}$ for images without motion blur. The cameras rotation is assumed to be at a maximum of 12/1000 degree (1/2 IFOV) during exposure time for a motion blur free image. The result is an error of 0.36 degree in the orientation between both cameras caused by the non-synchronous images.

For further processing a calibrated relative orientation of both cameras cannot be used.
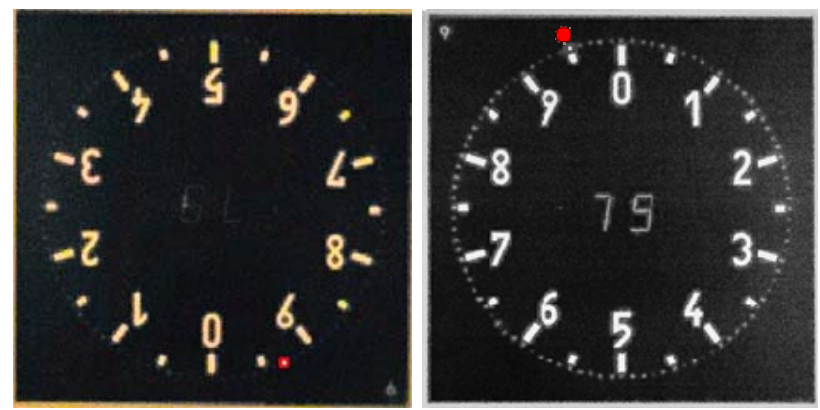

Figure 8. Investigation of simultaneous shutter release

\subsection{Data processing}

The data processing is done by projective transformation regarding the individual parameters of lens distortion. The transformation parameters must be calculated individually by identical points in two images for each image pair. As a result, a 4-channel image (fig. 9) will be generated containing the calibrated interior orientation parameters of the RGB camera. This can be used in a further photogrammetric evaluation process.

\subsection{Results}

For a first attempt, the same area as for the ADC Lite was covered and one image pair was evaluated. Compared to the Tetracam ADC Lite, an area can be represented with more details due to the better image quality of the Sigma DP1. 

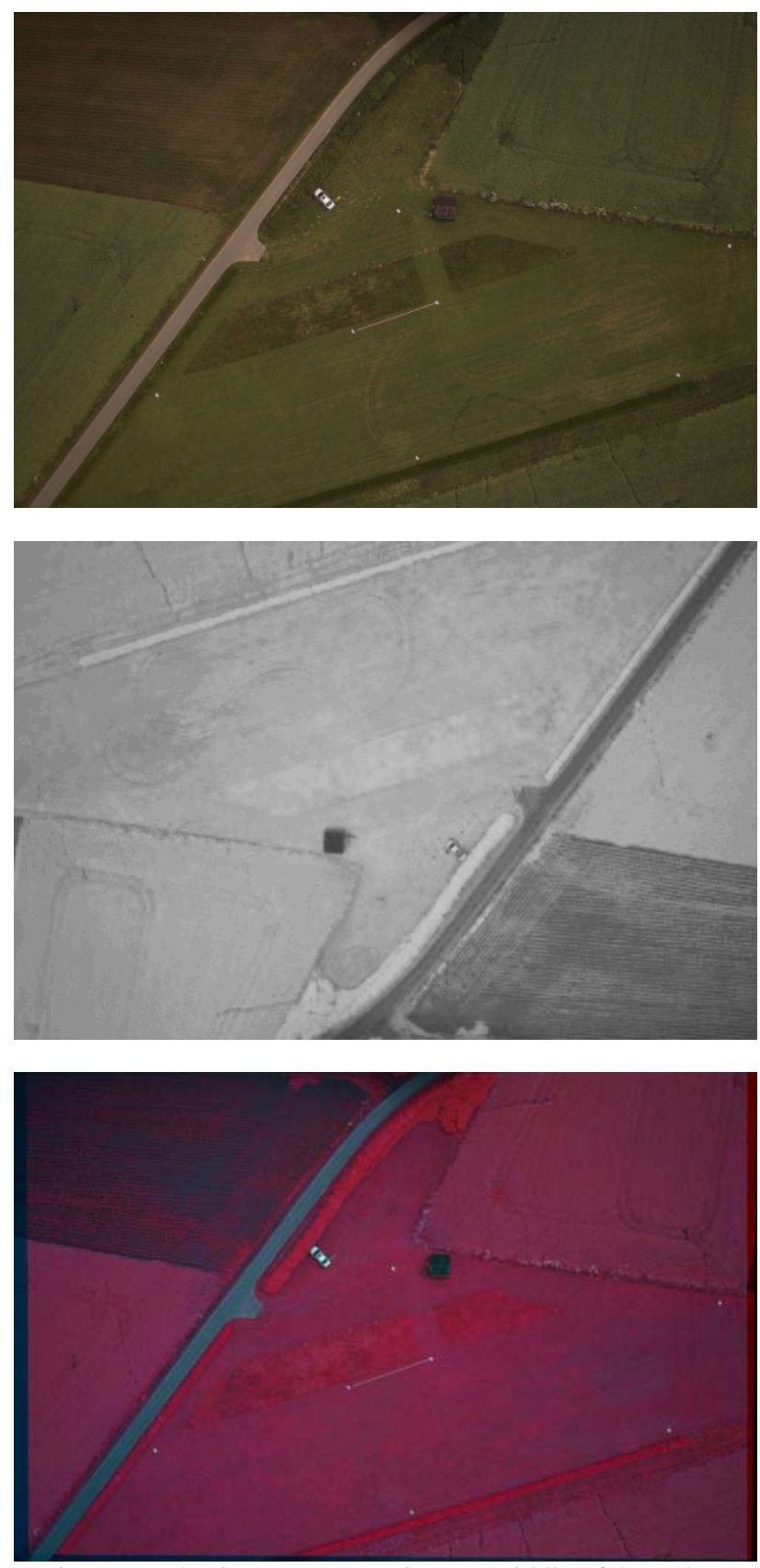

Figure 9. RGB image (top), IR image (middle), false colour image with green, red, infrared channel (down)

\section{SUMMARY}

The Tetracam ADC Lite is not suited for the use with the UAV MR-X8 presented in this paper due to a pronounced rolling shutter effect. The presented in-house developed sensor head containing two compact cameras Sigma DP1 is heavier ( $\sim 500$ g) than the ADC Lite (200 g), but still portable by the UAV. The sensor concept provides 4-channel image data with a blue, green, red (camera 1) and infrared channel (camera 2). The resulting image has a calibrated interior orientation for the use of conventional aerial photogrammetry software.

To obtain a geometric stability the cameras were focused in their respective spectral range on a typical flight altitude and all moving parts of the lens were glued. The stability has been verified by multiple calibrations.
If the images of both cameras are taken absolutely simultaneous, a calibrated relative orientation of both cameras can be used for the registration of the image layers. However, it was shown that the images have a delay up to $3 / 100 \mathrm{~s}$ despite the simultaneous triggering by an electric signal.

The geometric characteristics will be further observed during the upcoming projects. The radiometric characteristics of the sensor (spectral sensitivity) and other influences on the radiation-based measurements such as e.g. the modelling of BRDF (bidirectional reflectance distribution function) will be investigated in upcoming projects.

\section{REFERENCES}

Berni, J.A.J., Zarco-Tejada, P.J., Suárez, L., Gonzáles-Dugo, V., Fereres, E., 2009. Remote sensing of vegetation from UAV platforms using lightweight multispectral and thermal imaging sensors. In: The International Archives of the Photogrammetry, Remote Sensing and Spatial Information Sciences, Vol. XXXVIII-1-4-7/W5.

Gehrke, R., Greiwe, A., 2011. Sensoren zur kleinformatigen Aerophotogrammetrie mit UAV. In: DGPF Jahrestagung 2011, Publikationen der DGPF Band 20, pp. 75-82.

Geyer, C., Meingast, M., Sastry, S., 2005. Geometric models of rolling-shutter cameras. In: 6th OmniVis WS, University of California.

Gilblom, D. L., Yoo, S. K., Ventura, P., 2003. Operation and performance of a color image sensor with layered photodiodes. In: The Proceedings of the SPIE, Volume 5074, pp. 318-331.

Gilblom, D. L., Yoo, S., K., 2004. Infrared and ultraviolet imaging with a CMOS sensor having layered photodiodes. In: The Proceedings of the SPIE, Volume 5301, pp. 186-192.

Gini, R., Passoni, D., Pinto, L., Sona, G., 2012. Aerial Images from an UAV System: 3D Modelling and Tree Species Classification in a Park Area. In: The International Archives of the Photogrammetry, Remote Sensing and Spatial Information Sciences. Vol. XXXIX, Part B1, pp. 361-366.

Greiwe, A., Gehrke, R., 2013. Foveon Chip oder Bayer Pattern - Geeignete Sensoren zur Aerophotogrammetrie mit UAS. In: Oldenburger 3D Tage 2013, to be printed.

Huang, Y., Thomson, S. J., Lan, Y., Maas, S. J., 2010. Multispectral imaging systems for airborne remote sensing to support agricultural production management. Int J Agric \& Biol Eng, 2010; 3(1).

Knoth, C., Prinz, T. \& Loef, P., 2011. Microcopter-Based Color Infrared (CIR) Close Range Remote Sensing as a Subsidiary Tool for Precision Farming. In: Proceedings of the ISPRS Workshop on Methods for Change Detection and Process Modelling, Univ. Press Cologne, Cologne, Germany.

Nebiker, S., Annen, A., Scherrer, M., Oesch, D., 2008. A LightWeight Multispectral Sensor for Micro UAV - Opportunities for Very High Resolution Airborne Remote Sensing. In: The International Archives of the Photogrammetry, Remote Sensing 
and Spatial Information Sciences. Vol. XXXIIIX, Part B1, pp. 1193-1199.

Rieke-Zapp, D. H., Bommer-Dens, B., Ernst, D., 2010. Small Format Digital Photogrammetry for Applications in the Earth Sciences. In: The International Archives of the Photogrammetry, Remote Sensing and Spatial Information Sciences. Vol. XXXIII, Part 5, pp. 516 - 519.

Tetracam Inc., 2010. Agricultural Camera User’s Guide.

Verhoeven, G., 2010. It's all about the format - unleashing the power of RAW aerial photography. International Journal of Remote Sensing. Vol. 31,8, pp. 2009-2042.

Verhoeven, G., Smet, P., Poelman, D., Vermeulen, F., 2009. Spectral Characterization of a Digital Still Camera's NIR Modification to Enhance Archaeological Observation. In: IEEE Transactions on Geoscience and Remote Sensing, Volume 47 No. 10, pp. 3456 - 3468. 